\title{
Synchronizability of Two-Layer Cluster Ring Networks
}

\author{
Yang Deng, Zhen Jia*, Lin Liao \\ College of Science, Guilin University of Technology, Guilin, China \\ Email: ${ }^{*}$ jjjzzz0@163.com
}

How to cite this paper: Deng, Y., Jia, Z. and Liao, L. (2019) Synchronizability of Two-Layer Cluster Ring Networks. Communications and Network, 11, 35-51. https://doi.org/10.4236/cn.2019.112004

Received: March 1, 2019

Accepted: March 31, 2019

Published: April 3, 2019

Copyright $\odot 2019$ by author(s) and Scientific Research Publishing Inc. This work is licensed under the Creative Commons Attribution International License (CC BY 4.0).

http://creativecommons.org/licenses/by/4.0/

\begin{abstract}
Multilayer network is a frontier direction of network science research. In this paper, the cluster ring network is extended to a two-layer network model, and the inner structures of the cluster blocks are random, small world or scale-free. We study the influence of network scale, the interlayer linking weight and interlayer linking fraction on synchronizability. It is found that the synchronizability of the two-layer cluster ring network decreases with the increase of network size. There is an optimum value of the interlayer linking weight in the two-layer cluster ring network, which makes the synchronizability of the network reach the optimum. When the interlayer linking weight and the interlayer linking fraction are very small, the change of them will affect the synchronizability.
\end{abstract}

\section{Keywords}

Two-Layer Cluster Ring Network, Synchronizability, Interlayer Linking Weight, Interlayer Linking Fraction

\section{Introduction}

Complex networks are ubiquitous in the world, such as power and transportation networks, biological networks, economic and financial networks, and social networks. The study of complex networks in many disciplines has allowed us to better understand a myriad of complex phenomena, including the spread of disease on networks of human contacts, the functioning of intricate biological pathways, and gene circuits, as well as to provide theoretical support for engineers to control or optimize artificial interacting systems [1]-[6]. In the past 20 years, the synchronization problem of complex networks has attracted more and more attention from various disciplines [7]-[12] and has achieved many important research results. However, as the research deepens, people realize that many 
real-world networks do not exist in isolation. They are interrelated and constitute a larger and complex system called multilayer network. Examples of such networks are widespread; for instance, in a social system, a set of individuals interact between each other in various modes of social interactions between the same people: an individual has interactions with others through online social systems (such as Facebook or Twitter) and off-line systems (such as professional or personal circles). In another example, there are many forms of interaction between digital rumors, such as blogs, Tweets, and emails, not only on their respective networks, but also across each other. Therefore, multiple networks have attracted the interest of many researchers and become a new topic in network science research. In order to analyze the dynamic processes that appear in interactive networks, researchers have done a lot of research on multilayer networks. In 2013, Sole-Ribalta et al. extended the existing dynamical framework to deal with the dynamics of multilayer networks and analyzed the Laplacian spectrum behavior. Gomez et al. studied the time scales associated with diffusion processes that take place on a set of networks linked through interconnected layers and proposed the construction of supra-Laplacian matrices for the analysis of multiplex networks. In 2014, Lu et al. investigated the scalability of the synchronizability for ring or chain networks with dense clusters as the network size increases. In 2015, Xu et al. studied the synchronization of two-layer star networks. In 2008, Wei et al. analyzed the maximization of synchronization of two-layer networks, and analyzed the synchronization of two-layer networks composed of two randomly generated network layers with different interlayer node connection modes. It is found that the interlayer linking weight and the interlayer linking fraction have a profound influence on the synchronization of duplex networks [13]-[19].

Based on the above research, this paper extends the single-layer cluster ring networks studied by $\mathrm{Lu}$ et al. to a two-layer network model. In this paper, a two-layer cluster ring network model is proposed to study the effect of the change of the interlayer linking weight and linking fraction on the synchronizability of the two-layer cluster ring networks, and explores the change of synchronizability as the cluster blocks and nodes within the cluster blocks change.

This paper is organized as follows. Section 2 briefly introduces the master stability function (MSF) approach and network models used in this paper. Section 3 discusses how the route to synchronizability is affected by changing the network size for the complex cluster ring networks. Section 4 describes the relationship between the synchronizability and the interlayer linking weight and linking fraction. Finally, concluding comments are given in Section 5.

\section{The Model of Two-Layer Cluster Ring Network}

Cluster ring network refers to a network that connects $m$ cluster blocks into a ring [15] (which can be random, small world or scale-free network [14] in the cluster block). In this paper, we discuss the two-layer network with each layer 
being cluster ring, the number of nodes and cluster blocks in each layer is the same, but the structure of each layer can be different, and each layer network has its own topology structure formed by the connection within the layer. Intralayer connections can be directed or undirected, weighted or unweighted. Interlayer connections are channels for communicating different layers, or they can be directed or undirected, weighted or unweighted. When intralayer connections and interlayer connections are given, the structure of the multilayer network can be determined [16] [17]. Figure 1 is a schematic diagram of a simple two-layer cluster ring network, in which nodes of each layer are connected by a interlayer linking fraction $\gamma=1$.

For a multiplex network consisting of $M$ layers each consisting of $N$ nodes, the dynamics of $n$-dimensional node $x_{i}^{\alpha}$ (the $i$-th node in the $\alpha$-th layer) can be described by the following differential equation [18]:

$$
\frac{\mathrm{d} x_{i}^{\alpha}}{\mathrm{d} t}=f_{i}^{\alpha}\left(x_{i}^{\alpha}\right)+\sum_{j=1}^{N} a_{i j}^{\alpha} \Gamma_{1}\left(x_{j}^{\alpha}-x_{i}^{\alpha}\right)+\sum_{\beta=1}^{M} \omega_{i}^{\alpha \beta} \Gamma_{2}\left(x_{i}^{\beta}-x_{i}^{\alpha}\right),
$$

where $1 \leq i \leq N, 1 \leq \alpha \leq M, \quad x_{i}^{a} \in \mathfrak{R}^{n}$ is the state vector of the $i$-th node in the $\alpha$-th layer, $f_{i}^{\alpha}: \mathfrak{R}^{n} \rightarrow \Re^{n}$ governs the dynamics of the $i$-th node in the $\alpha$-th layer, $\Gamma_{1}: \Re^{n} \rightarrow \mathfrak{R}^{n}$ is the inner coupling function defining the interaction between nodes within any particular layer, and $\Gamma_{2}: \mathfrak{R}^{n} \rightarrow \mathfrak{R}^{n}$ is the inner coupling function defining the interaction between nodes on separate layers. To apply the master stability framework to the composite multiplex, it is necessary to assert identical nodal dynamics and identical coupling functions: $f_{i}^{\alpha}=f$ and $\Gamma_{1}=\Gamma_{2}=\Gamma$. The intralayer linking weight $a_{i j}^{\alpha}$ is positive if and only if there is a link from node $j$ to node $i(j \neq i)$ within the $\alpha$-th layer. Otherwise, $a_{i j}^{\alpha}=0$. The interlayer linking weight $\omega_{i}^{\alpha \beta}$ is similarly positive if and only if there is a link between node $i$ in layer $\alpha$ and node $i$ in layer $\beta$. Otherwise, $\omega_{i}^{\alpha \beta}=0$. Note that there are no links between node $i$ on layer $\alpha$ and a different node $j(j \neq i)$ on a different layer $\beta \quad(\alpha \neq \beta)$.

Two-layer cluster ring network dynamics model is as follows:

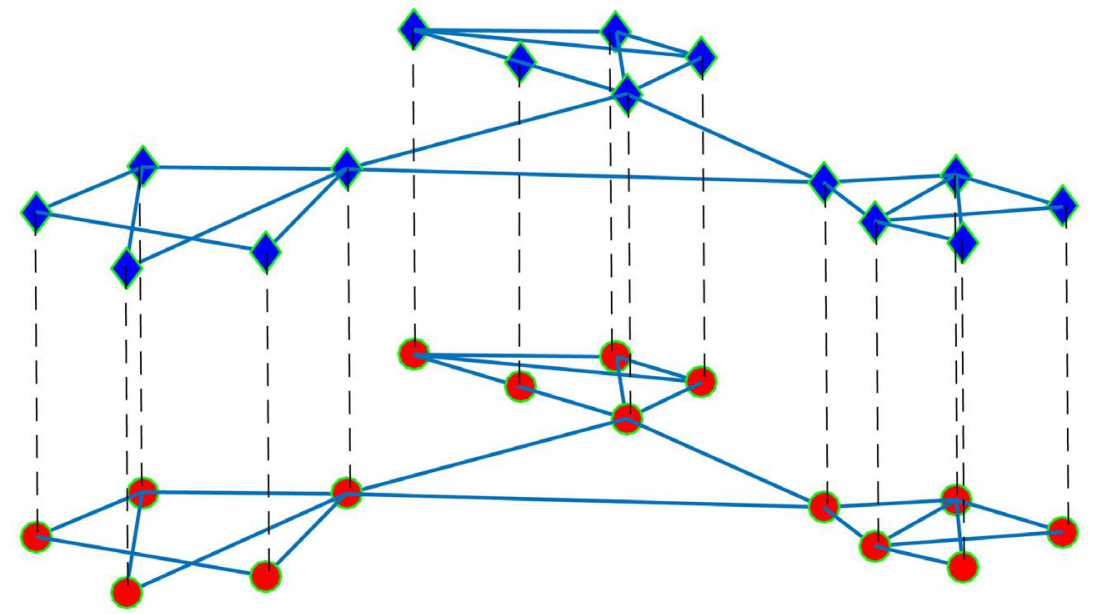

Figure 1. schematic diagram of two-layer clustering ring network. 


$$
\frac{\mathrm{d} x_{i}^{\alpha}}{\mathrm{d} t}=f_{i}^{\alpha}\left(x_{i}^{\alpha}\right)+\sum_{j=1}^{N} a_{i j}^{\alpha} \Gamma_{1}\left(x_{j}^{\alpha}-x_{i}^{\alpha}\right)+\sum_{\beta=1}^{2} \omega_{i}^{\alpha \beta} \Gamma_{2}\left(x_{i}^{\beta}-x_{i}^{\alpha}\right),
$$

where $1 \leq i \leq N, 1 \leq \alpha \leq 2$.

Corresponding to a network, the eigenvalues of Laplacian matrices are very important for studying network dynamics. A Laplacian matrix of a multilayer network is called a super-Laplacian matrix $£$, it can be decomposed into two parts: the intralayer super-Laplacian matrix $£^{L}$ and the interlayer super-Laplacian matrix $£^{I} \quad[19]$ :

$$
£=£^{L}+£^{I}
$$

As for $£^{L}$, it can be represented by the direct sum of the Laplacian matrix within each layer, namely,

$$
£^{L}=\left(\begin{array}{cccc}
a_{1} L^{(1)} & 0 & \cdots & 0 \\
0 & a_{2} L^{(2)} & \cdots & 0 \\
\vdots & \vdots & \ddots & \vdots \\
0 & 0 & \cdots & a_{M} L^{(M)}
\end{array}\right)=\bigoplus_{\alpha=1}^{M} a_{\alpha} L^{(\alpha)}
$$

where $a_{\alpha}$ is the intralayer linking weight in the $\alpha$-layer. $L_{(i, j)}^{(\alpha)}=-1$, if there is a link from node $j$ to node $i$ in the $\alpha$-th layer. Otherwise, $L_{(i, j)}^{(\alpha)}=0$, and all rows of $L^{(\alpha)}$ sum up to 0 . For a two-layer network, when the interlayer linking weight is $\omega$, the interlayer super-Laplacian matrix $£^{I}$ is:

$$
£^{I}=L^{I} \otimes I=\left(\begin{array}{cc}
\omega & -\omega \\
-\omega & \omega
\end{array}\right) \otimes I=\left(\begin{array}{cc}
\omega I & -\omega I \\
-\omega I & \omega I
\end{array}\right)
$$

For a two-layer network with $N$ nodes per layer, the super-Laplacian matrix is

$$
\begin{aligned}
£ & =£^{L}+£^{I}=\left(\begin{array}{cc}
a_{1} L^{(1)} & 0 \\
0 & a_{2} L^{(2)}
\end{array}\right)+\omega\left(\begin{array}{cc}
I & -I \\
-I & I
\end{array}\right) \\
& =\left(\begin{array}{cc}
a_{1} L^{(1)}+\omega I & -\omega I \\
-\omega I & a_{2} L^{(2)}+\omega I
\end{array}\right)
\end{aligned}
$$

where $\omega$ is the interlayer linking weight, $a_{1}$ and $a_{2}$ are the intralayer linking weight in the first and second layers, respectively. $L^{(1)}$ and $L^{(2)}$ are the Laplacian matrix in the first and second layers, respectively. $I$ is the $N \times N$ identity matrix.

\section{Scale Variability of Synchronizability}

Consider a general complex cluster network with $m$ clusters, where each cluster contains $n$ nodes and all clusters arrange into a ring. The linking between neighboring clusters is specific intercluster linking, involving selecting a specific node from each cluster. The cluster block is composed of random, small world or scale-free structures. In this paper, we discuss the change of synchronizability of two-layer cluster ring network when the network size increases. The increase in the size of the network can be divided into two situations: the first case is to keep the cluster block of the two-layer cluster ring network unchanged $(m=20)$, 
and increase the number of nodes in each block ( $n$ from 10 to 100), the numerical simulation results are shown in Figure 2. The second case is to keep the number of nodes within each block unchanged $(n=20)$, and increases the number of blocks of cluster rings ( $m$ from 10 to 100 ), the numerical simulation results are shown in Figure 3. In each experiment, we take the average of the results of 50 tests, $\lambda_{2}$ and $R=\lambda_{N} / \lambda_{2}$ will be used to investigate the scalability of network synchronizability in this paper.

Figure 2 shows $\lambda_{2}$ and $\lambda_{N} / \lambda_{2}$ for network with $m=20$ as $n$ increases from 10 to 100 . The indication is that the synchronizability of network decreases monotonically with increasing size $n$. Figure 3 shows $\lambda_{2}$ and $\lambda_{N} / \lambda_{2}$ for network with $n=20$ as $m$ increases from 10 to 100 . The indication is that the synchronizability of network decreases monotonically with increasing size $\mathrm{m}$. In fact, the two-layer cluster ring network and the single-layer cluster ring network

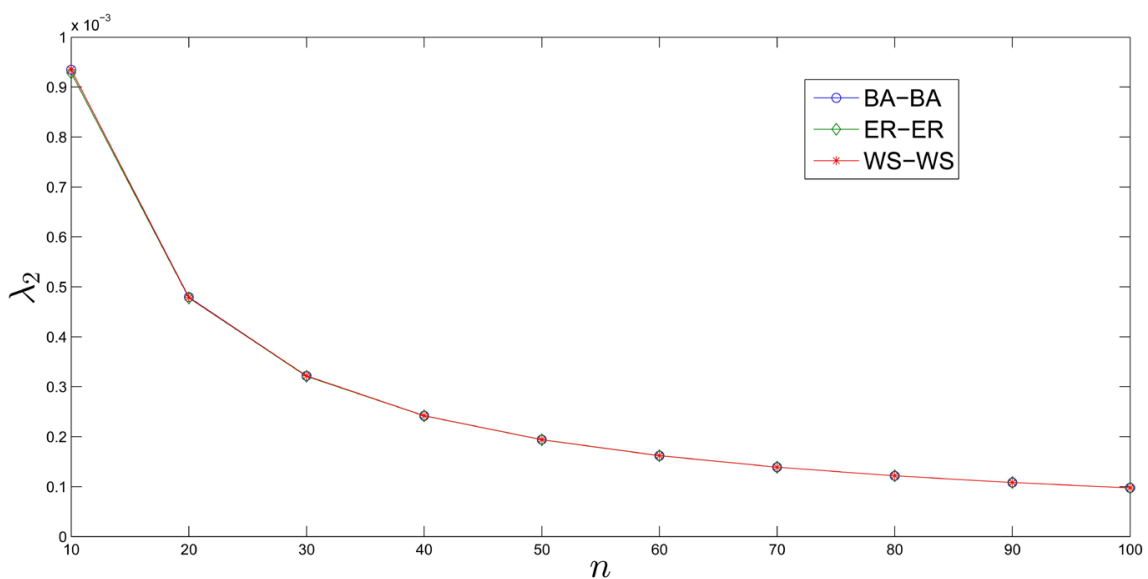

(a)

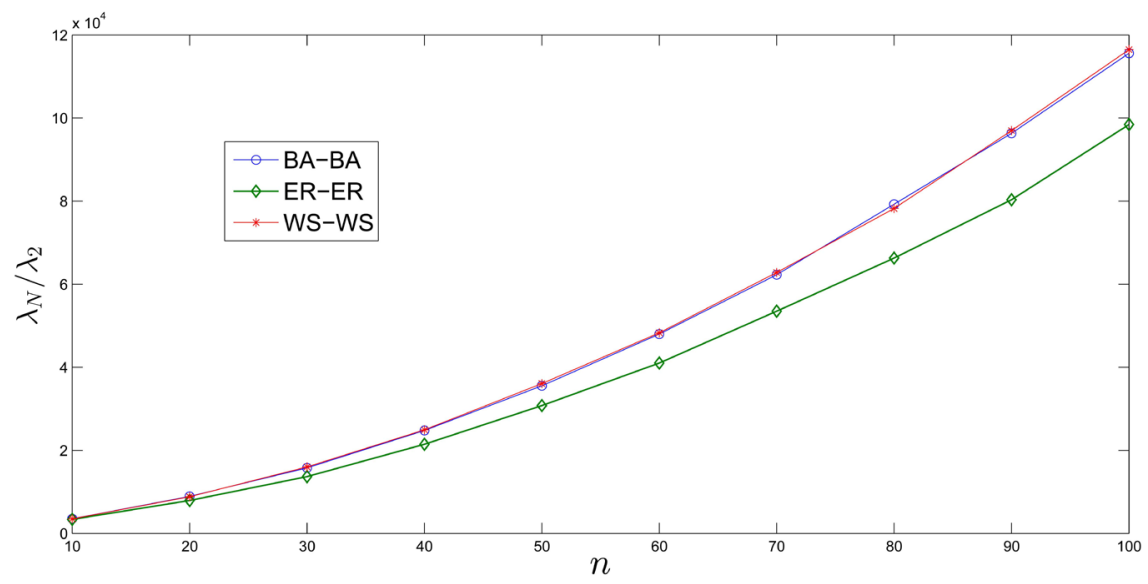

(b)

Figure 2. (a) $\lambda_{2}$ versus $n$ with $m=20$, (b) $\lambda_{N} / \lambda_{2}$ versus $n$ with $m=20$. Blue circular curves (BA-BA): the internal structure of the cluster blocks in the two-layer cluster ring network is scale-free; green diamond curves (ER-ER): the internal structure of the cluster blocks in the two-layer cluster ring network is random; red star curves (WS-WS): the internal structure of the cluster blocks in the two-layer cluster ring network is small-world. 


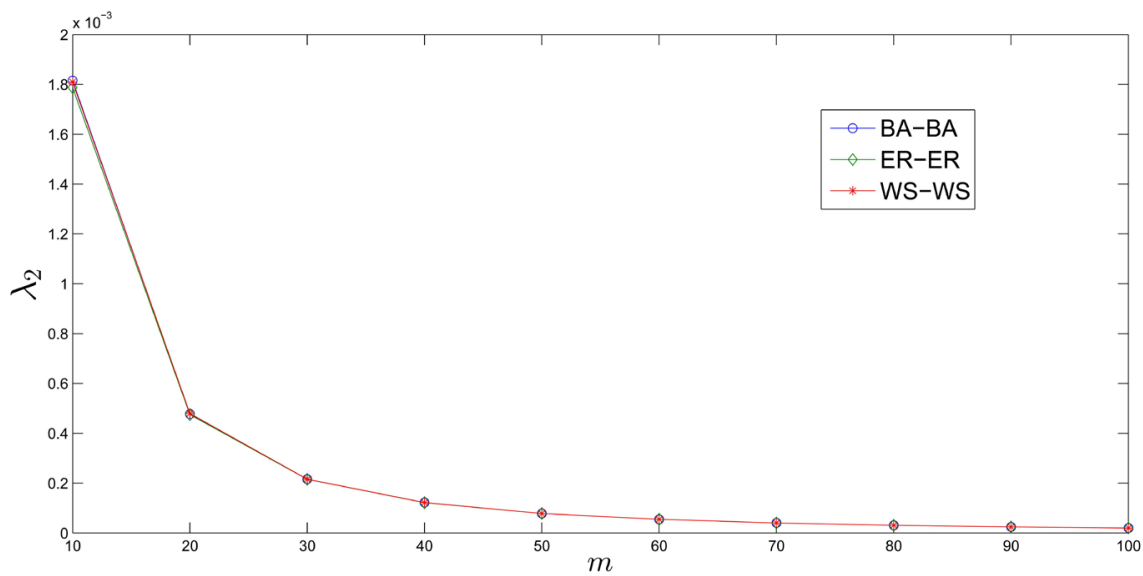

(a)

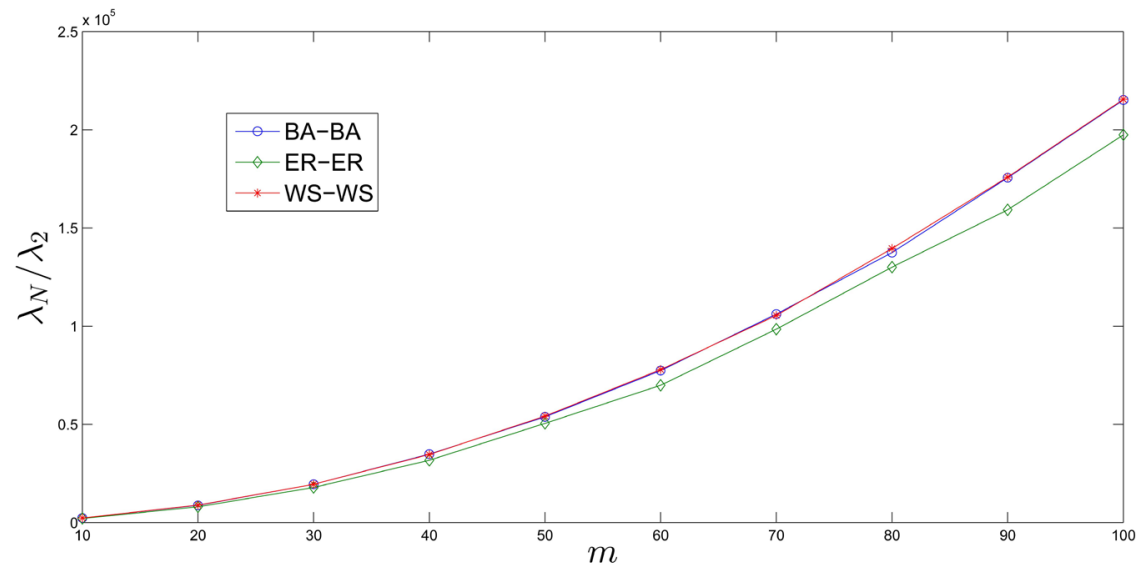

(b)

Figure 3. (a) $\lambda_{2}$ versus $m$ with $n=20$, (b) $\lambda_{N} / \lambda_{2}$ versus $m$ with $n=20$. Blue circular curves (BA-BA): the internal structure of the cluster blocks in the two-layer cluster ring network is scale-free; green diamond curves (ER-ER): the internal structure of the cluster blocks in the two-layer cluster ring network is random; red star curves (WS-WS): the internal structure of the cluster blocks in the two-layer cluster ring network is small-world.

[15] have the same simulation results in terms of the scalability of synchronizability. Whether it is a cluster ring network with a fixed number of clusters or a cluster ring network with a fixed number of nodes in the cluster are not scalable with respect to synchronizability. The synchronizability is basically independent of the random, small world, and scale-free structure inside the cluster block, and the synchronizability is sensitive to the changes in networks size (the change of cluster blocks and nodes in cluster blocks).

\section{Influence of Network Structure Parameters on Synchronizability}

We consider the impact of the interlayer linking weight and interlayer linking fraction on synchronizability of two-layer cluster ring network. Each layer nodes of the network is fixed to $N=1000$. We recorded $\lambda_{2}$ and $\log _{2} R$ of the Lap- 
lacian matrix obtained by running 50 times for each network model. The experimental simulation data was taken as the average of 50 experiments.

\subsection{Synchronizability versus Interlayer Linking Weight}

First, we consider the impact of the interlayer linking weight on network synchronizability. We assume that the interlayer linking fraction $\gamma=1$, that is, every node in one layer is linked to its counterpart in the other layer. The interlayer linking weight $\omega$ is varied from 0.0002 to 2.002 . The simulation results are shown in Figure 4.

Figure 4(a) \& Figure 4(b) display the smallest nonzero eigenvalue $\lambda_{2}$ and $\log$-eigenratio $\log _{2} R$ with respect to varying values of the interlayer linking weight $\omega$ ( $\omega$ from 0.0002 to 0.02 ), respectively. The subgraph of Figure 4(a) \& Figure 4(b) displays the smallest nonzero eigenvalue $\lambda_{2}$ and log-eigenratio $\log _{2} R$ with respect to varying values of the interlayer linking weight $\omega(\omega$ from 0.002 to 2.002 ), respectively.

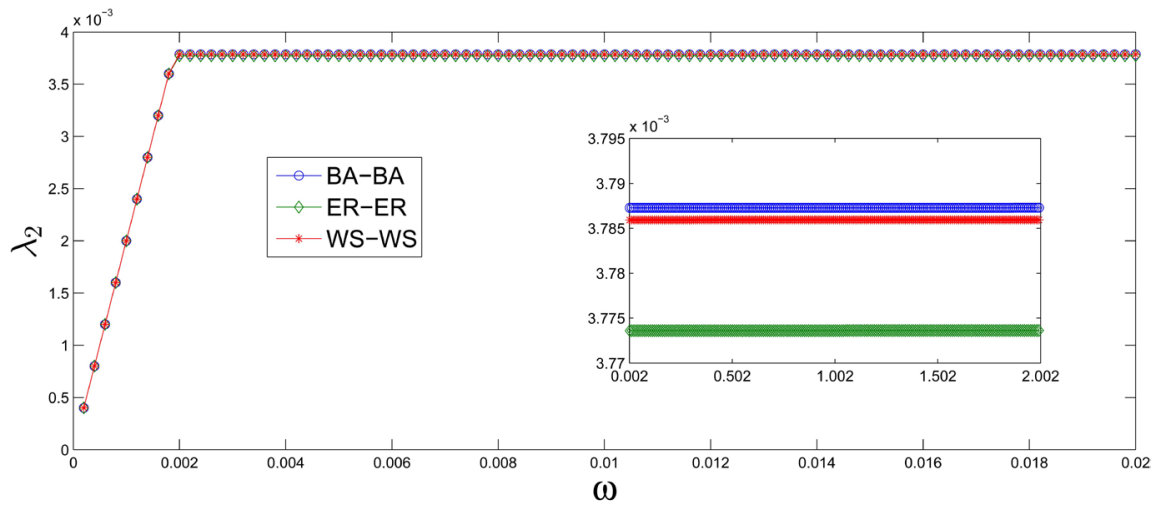

(a)



(b)

Figure 4. (a) $\lambda_{2}$ with respect to the interlayer linking weight $\omega$, with interlayer linking fraction $\gamma=1$. (b) $\log$-eigenratio $\log _{2} R$ with respect to the interlayer linking weight $\omega$, with interlayer linking fraction $\gamma=1$. Blue circular curves (BA-BA): the internal structure of the cluster blocks in the two-layer cluster ring network is scale-free; green diamond curves (ER-ER): the internal structure of the cluster blocks in the two-layer cluster ring network is random; red star curves (WS-WS): the internal structure of the cluster blocks in the two-layer cluster ring network is small-world. 
Figure 4 shows when the interlayer linking weight $\omega$ from 0.0002 to 0.002 , the smallest nonzero eigenvalue $\lambda_{2}$ of the Laplacian matrix increases sharply, the log-eigenratio $\log _{2} R$ drops sharply, and the network synchronizability is strengthened. This means that when the interlayer linking weight is small, it can cause dramatic changes in $\lambda_{2}$ and $\log _{2} R$. The interlayer linking weight $\omega$ increases from 0.002 to $2.002, \lambda_{2}$ of the Laplacian matrix remain substantially unchanged, but, $\log _{2} R$ of the Laplacian matrix shows a small increase. So, when the two-layer cluster ring network has a fixed interlayer linking fraction between layers, as the interlayer linking weight of the network increases, the smallest nonzero eigenvalue $\lambda_{2}$ increases first and then remains stable, the $\log$-eigenratio $\log _{2} R$ is significantly reduce and then slowly increase. There is an optimal the interlayer linking weight value to optimize the network synchronizability. Whether the cluster ring is random, small world or scale-free, the synchronizability changes with the same trend.

\subsection{Synchronizability versus Interlayer Linking Fraction}

Now, we explore the effect of interlayer linking fraction on synchronizability. The interlayer linking weight $(\omega=1)$ is fixed, we randomly connect the node pairs in different layers and change the connection probability. The interlayer linking fraction $\gamma$ is varied from 0.001 to 1 . The simulation results are shown in Figure 5.

Figure 5(a) shows the interlayer linking weight $(\omega=1)$ is fixed, the the smallest nonzero eigenvalue $\lambda_{2}$ of the Laplacian matrix remains basically unchanged with the increase of the interlayer linking fraction. Figure 5(b) shows the log-eigenratio $\log _{2} R$ of the Laplacian matrix increases slightly with the increase of the interlayer linking fraction.

\subsection{Phase Diagrams for Synchronizability}

Phase diagrams for $\lambda_{2}$ and $\log _{2} R$ with respect to both $\omega$ and $\gamma$ are displayed in Figures 6-11 to illustrate the impact of the two parameters on the



(a) 


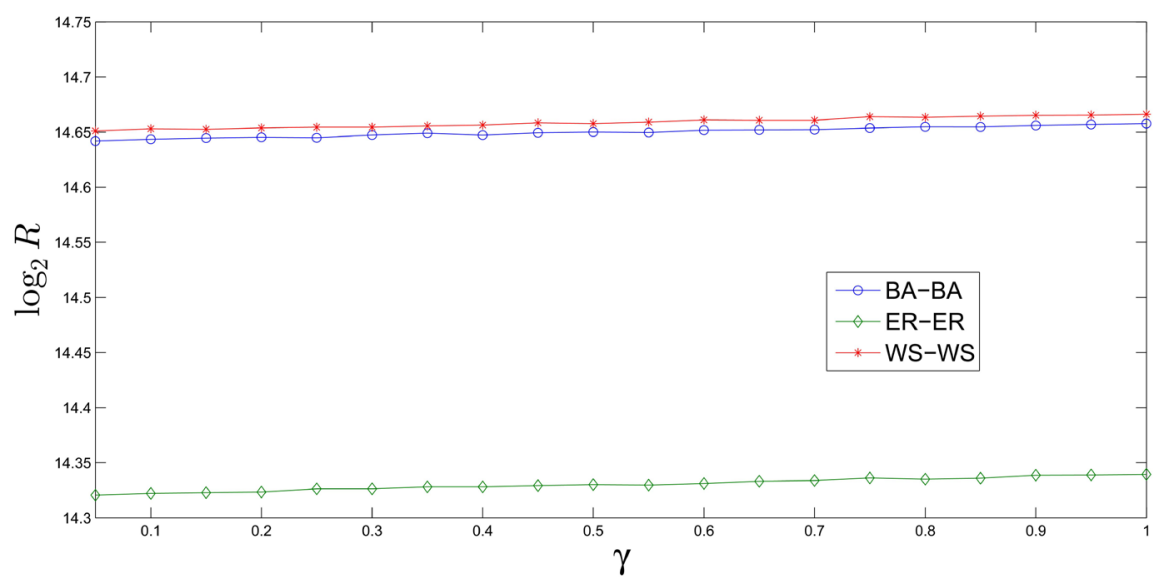

(b)

Figure 5. (a) $\lambda_{2}$ with respect to the interlayer linking fraction $\gamma$, with interlayer linking weight $\omega=1$. (b) log-eigenratio $\log _{2} R$ with respect to the interlayer linking fraction $\gamma$, with interlayer linking weight $\omega=1$. Blue circular curves (BA-BA): the internal structure of the cluster blocks in the two-layer cluster ring network is scale-free; green diamond curves (ER-ER): the internal structure of the cluster blocks in the two-layer cluster ring network is random; red star curves (WS-WS): the internal structure of the cluster blocks in the two-layer cluster ring network is small-world.



(a)

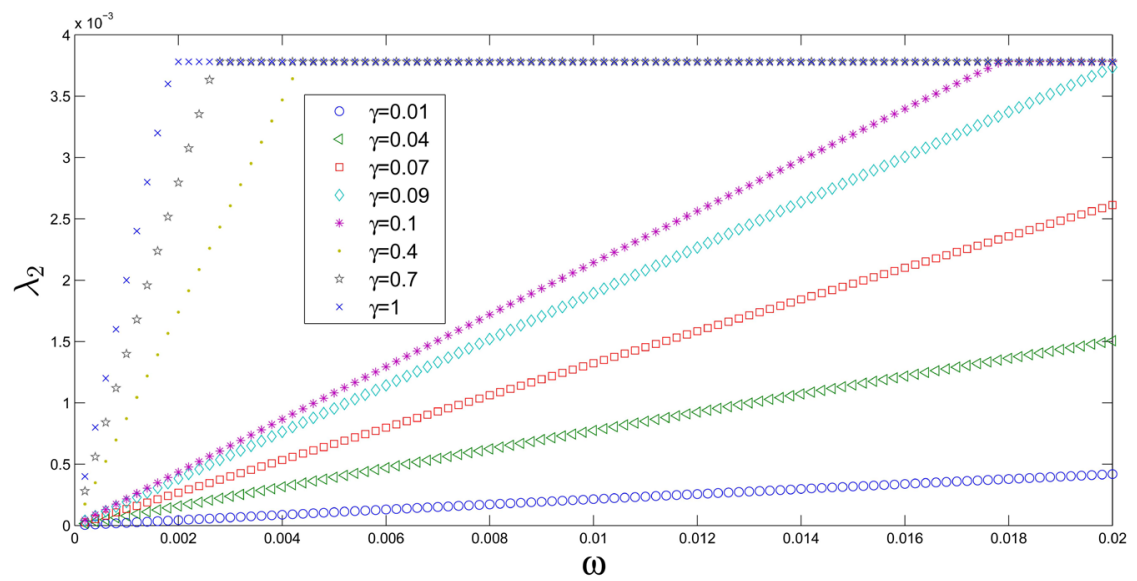

(b) 


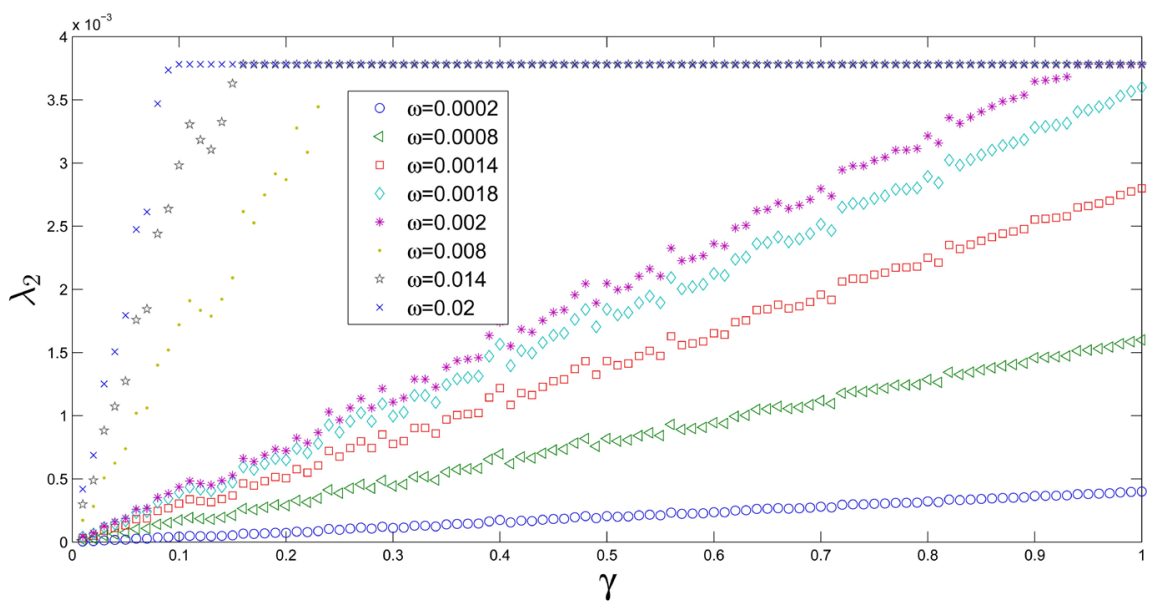

(c)

Figure 6. (a) Phase diagram for $\lambda_{2}$ in the two-dimensional parameter space $(\omega, \gamma)$. (b) a $\lambda_{2}$ variation diagram of a two-layer BA-cluster ring network with respect to $\omega$. (c) a $\lambda_{2}$ variation diagram of a two-layer BA-cluster ring network with respect to $\gamma$.

synchronizability of two-layer cluter ring networks, for the three different two-layer network models. $\omega$ takes a change between 0.0002 and 0.02 , and $\gamma$ takes a change from 0.01 to 1 . BA-cluster ring network denotes that the internal structure of the cluster blocks in the two-layer cluster ring network is scale-free. ER-cluster ring network denotes that the internal structure of the cluster blocks in the two-layer cluster ring network is random. WS-cluster ring network denotes that the internal structure of the cluster blocks in the two-layer cluster ring network is small-world.

Figure $6(\mathrm{a})$ is phase diagrams for $\lambda_{2}$ in the two-dimensional parameter space $(\omega, \gamma)$ for randomly correlated two-layer BA-cluster ring networks. Figure 6(b) is the variation of $\lambda_{2}$ of the network corresponding to different $\gamma$ values with $\omega$. Figure $6(\mathrm{c})$ is the variation of $\lambda_{2}$ of the network corresponding to different $\omega$ values with $\gamma$. Figure 6(a) \& Figure 6(b) show when the interlayer linking fraction $\gamma \in[0.01,0.09], \lambda_{2}$ increases as the interlayer linking weight increases, and the network synchronizability increases as the interlayer linking weight increases. When the interlayer linking fraction $\gamma \in[0.1,1]$, $\lambda_{2}$ increases first and then remains stable as the interlayer linking weight increases. Network synchronizability enhances first and remains unchange. Figure 6(a) \& Figure 6(c) show when the interlayer linking weight $\omega \in[0.0002,0.0018]$, $\lambda_{2}$ increases as the interlayer linking fraction increases, and the network synchronizability increases as the interlayer linking fraction increases. When the interlayer linking weight $\omega \in[0.002,0.02], \lambda_{2}$ increases first and then remains stable as the interlayer linking weight increases. Network synchronizability enhances first and remains unchange.

In conclusion, we find that $\omega(\omega \in[0.0002,0.0018])$ is maintained very small, synchronizability is greatly affected by $\gamma$, and the synchronizability of the network will increase as $\gamma$ increases. When $\omega(\omega \in[0.002,0.02])$ is slightly 




(a)

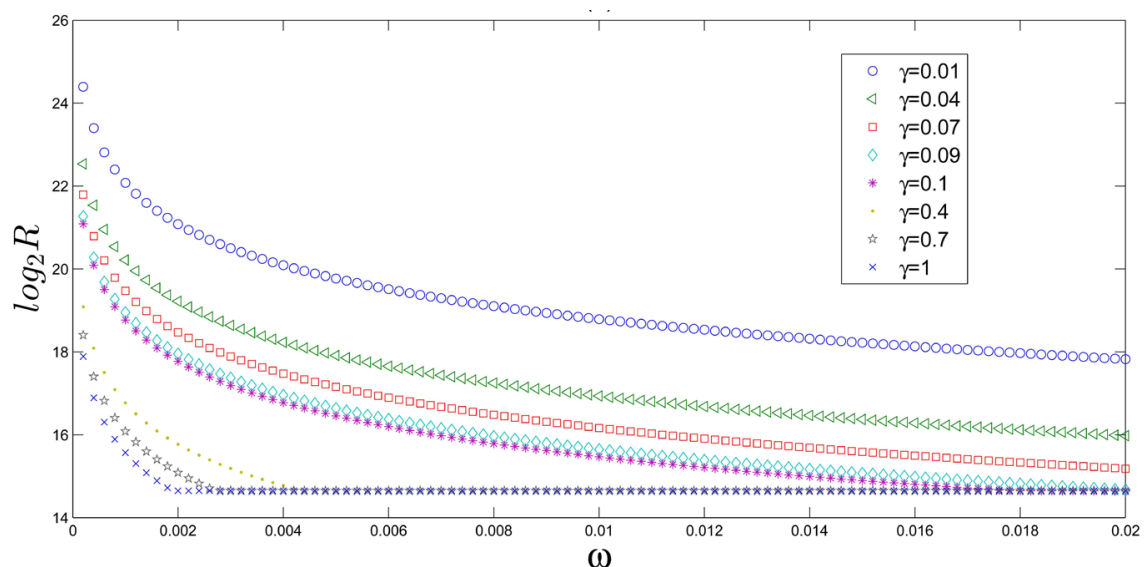

(b)

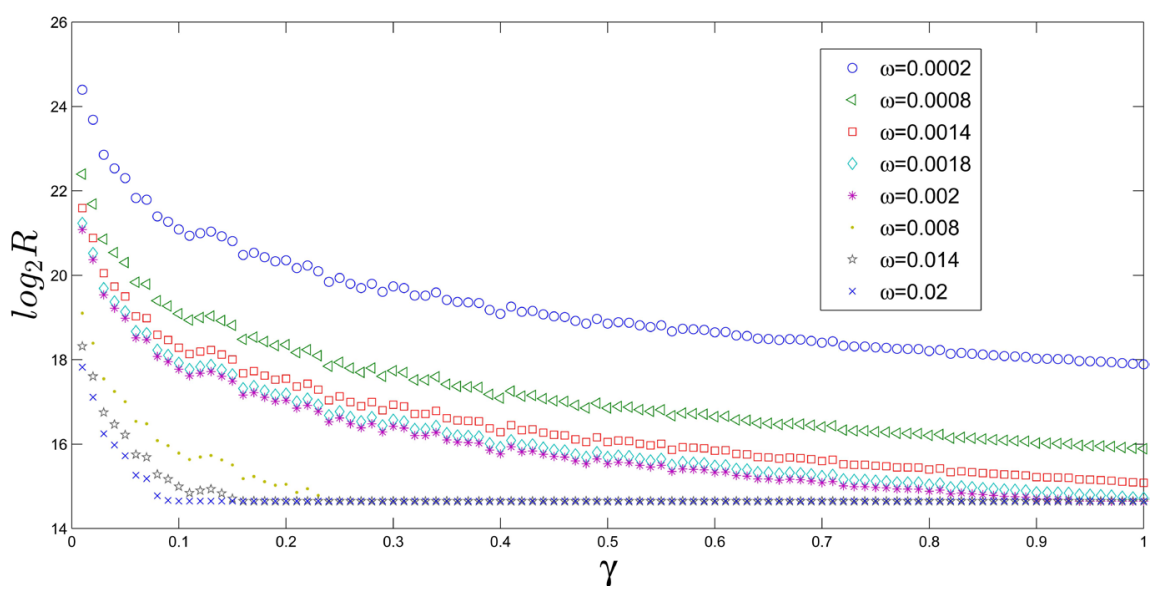

(c)

Figure 7. (a) Phase diagram for $\log _{2} R$ in the two-dimensional parameter space $(\omega, \gamma)$. (b) a $\log _{2} R$ variation diagram of a two-layer BA-cluster ring network with respect to $\omega$. (c) a $\log _{2} R$ variation diagram of a two-layer BA-cluster ring network with respect to $\gamma$.

larger, $\gamma$ is getting bigger and bigger, and the synchronizability is not affected by $\gamma \cdot \gamma(\gamma \in[0.01,0.09])$ is maintained very small, synchronizability is greatly 




(a)

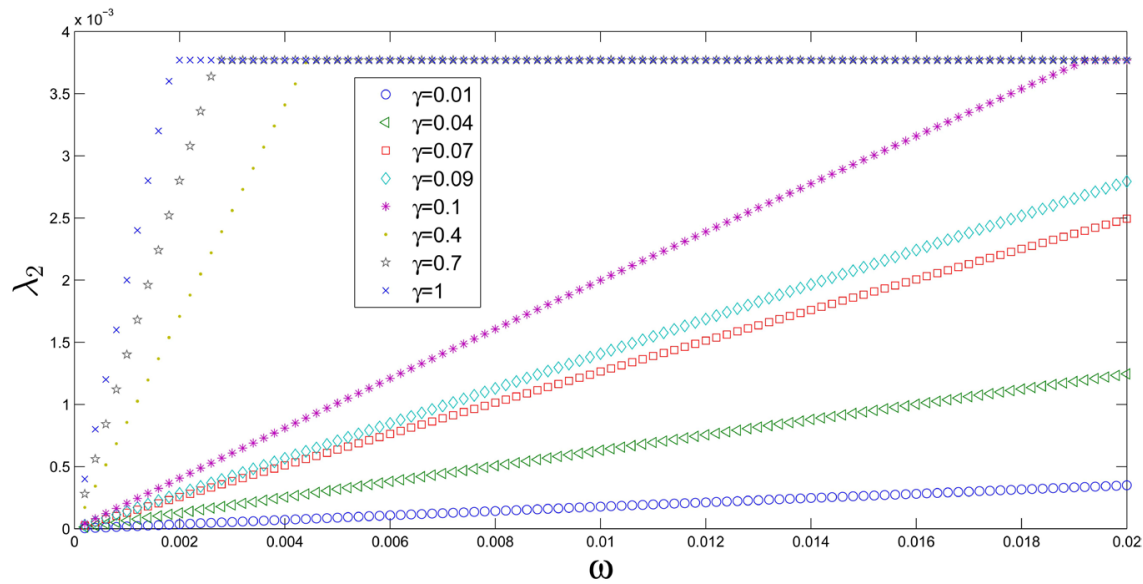

(b)

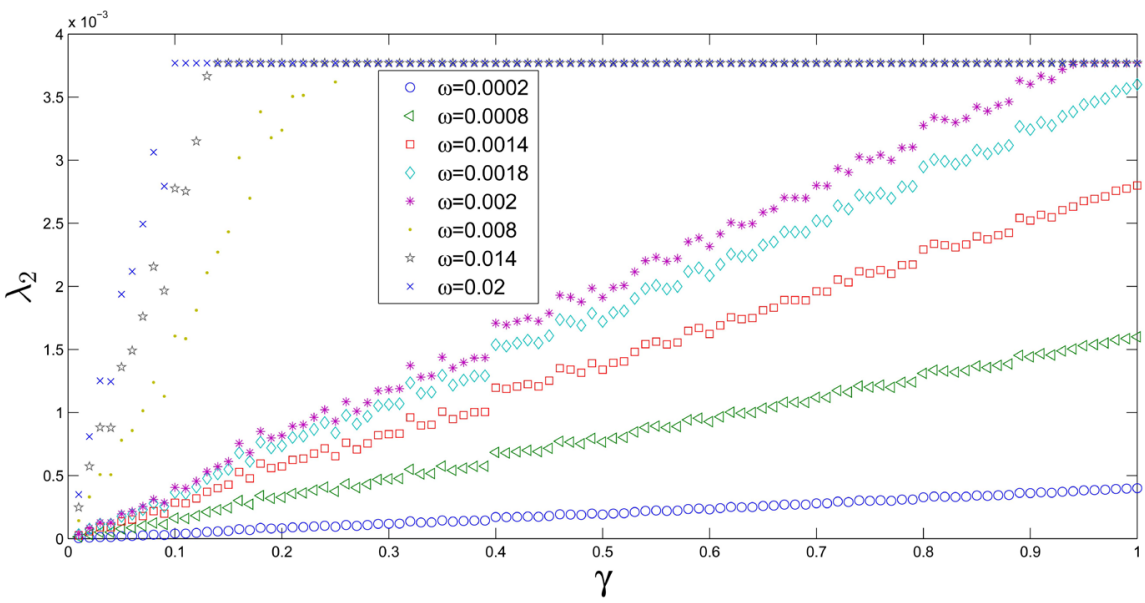

(c)

Figure 8. (a) Phase diagram for $\lambda_{2}$ in the two-dimensional parameter space $(\omega, \gamma)$. (b) a $\lambda_{2}$ variation diagram of a two-layer ER-cluster ring network with respect to $\omega$. (c) a $\lambda_{2}$ variation diagram of a two-layer ER-cluster ring network with respect to $\gamma$.

affected by $\omega$, and the synchronizability of the network will increase as $\omega$ increases. When $\gamma \quad(\gamma \in[0.1,1])$ is slightly larger, $\omega$ is getting bigger and big- 
ger, and the synchronizability is not affected by $\omega$.

Figure 7(a) is phase diagrams for $\log _{2} R$ in the two-dimensional parameter

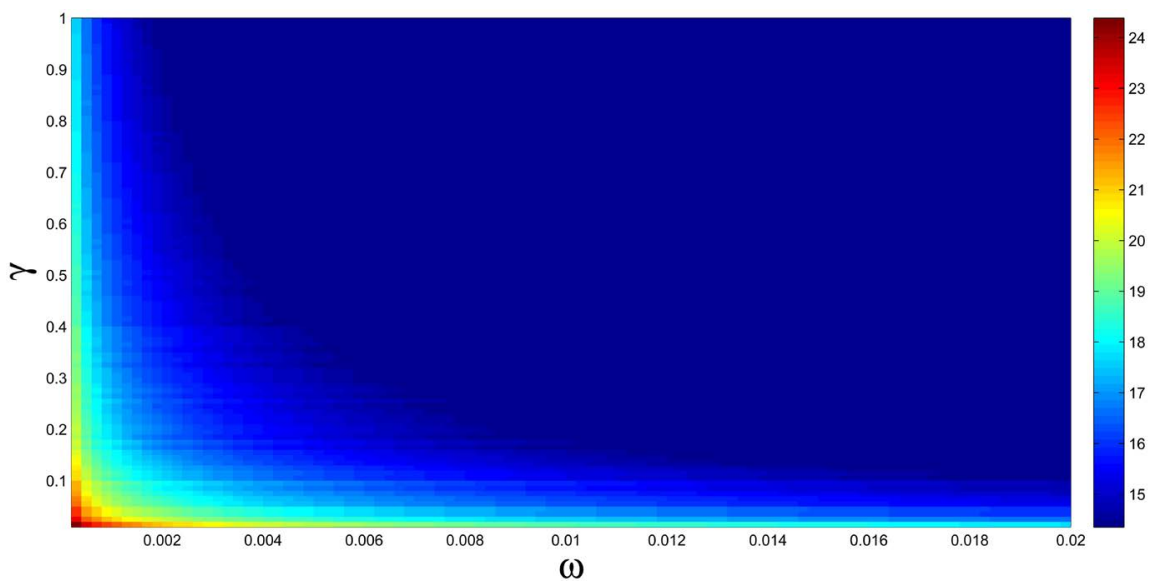

(a)

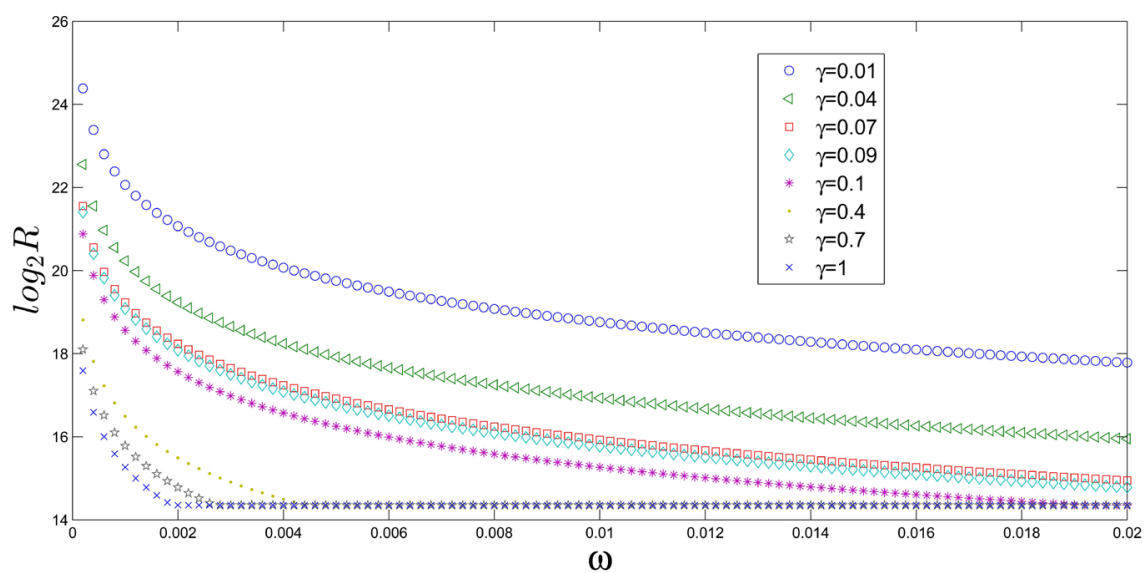

(b)

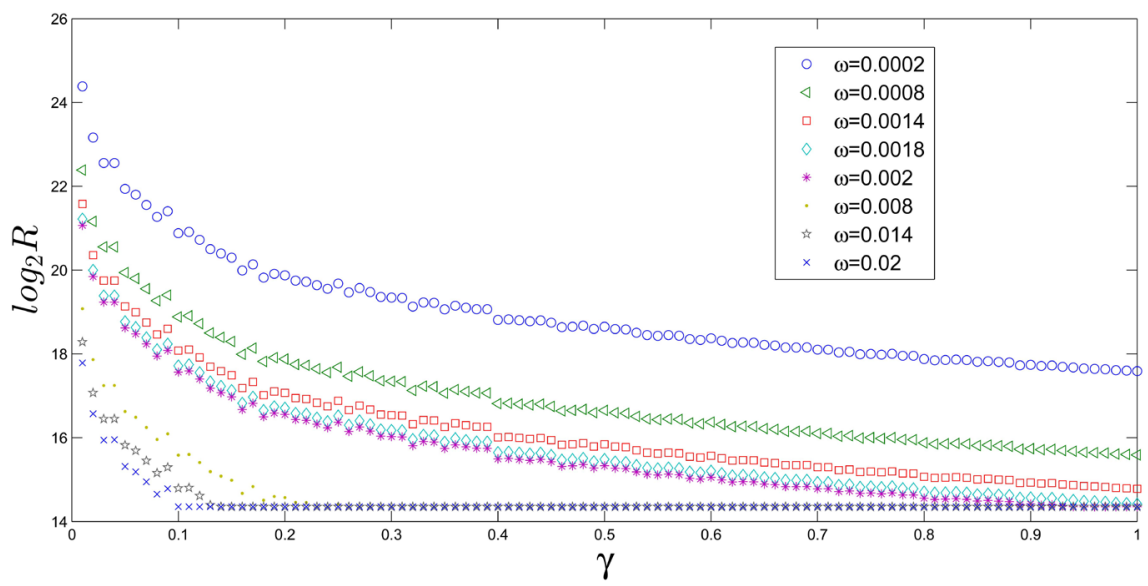

(c)

Figure 9. (a) Phase diagram for $\log _{2} R$ in the two-dimensional parameter space $(\omega, \gamma)$. (b) a $\log _{2} R$ variation diagram of a two-layer ER-cluster ring network with respect to $\omega$. (c) a $\log _{2} R$ variation diagram of a two-layer ER-cluster ring network with respect to $\gamma$. 




(a)

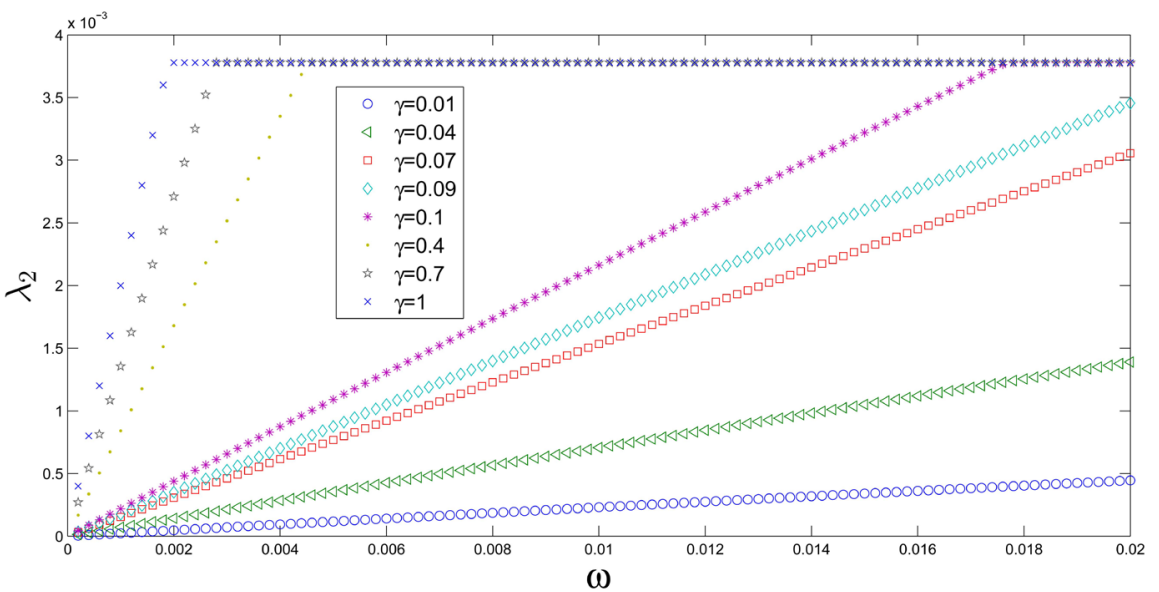

(b)

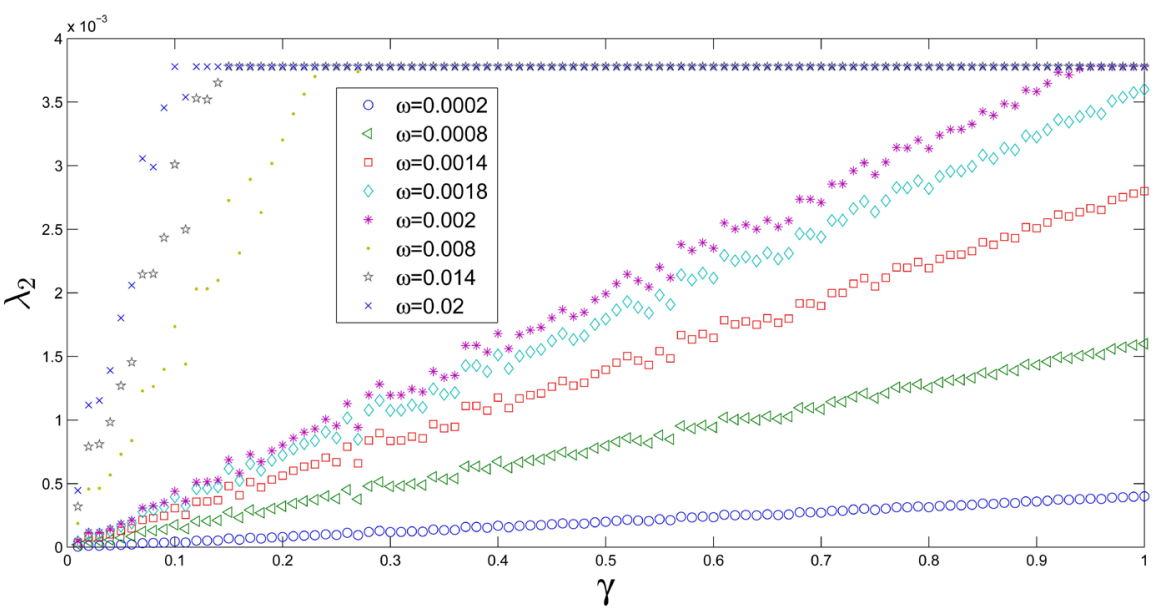

(c)

Figure 10. (a) Phase diagram for $\lambda_{2}$ in the two-dimensional parameter space $(\omega, \gamma)$. (b) a $\lambda_{2}$ variation diagram of a two-layer WS-cluster ring network with respect to $\omega$. (c) a $\lambda_{2}$ variation diagram of a two-layer WS-cluster ring network with respect to $\gamma$.

space $(\omega, \gamma)$ for randomly correlated two-layer BA-cluster ring networks. Figure 7 (b) is the variation of $\log _{2} R$ of the network corresponding to different 


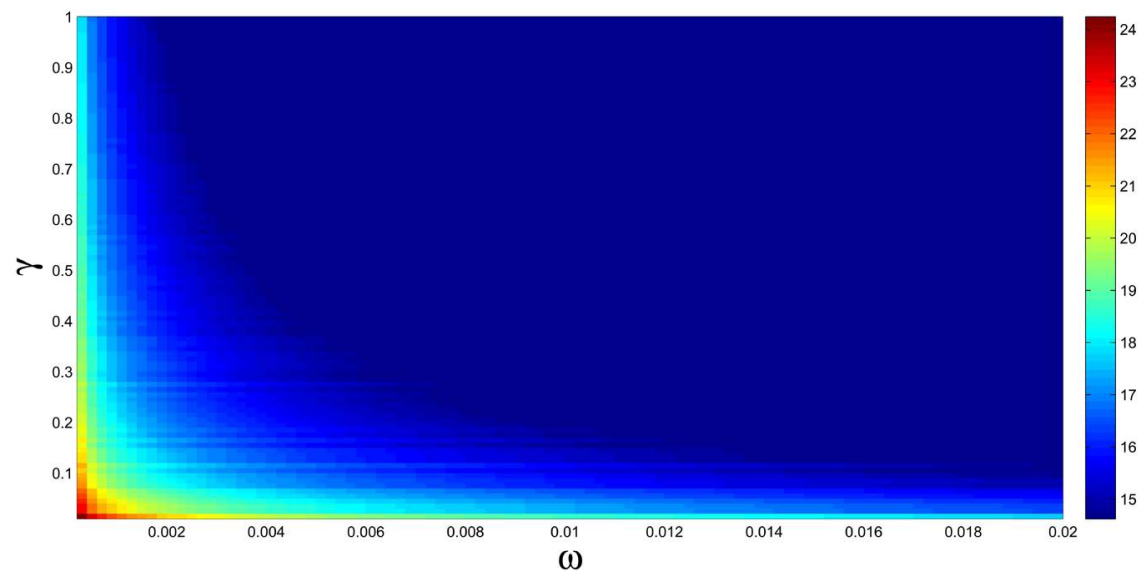

(a)

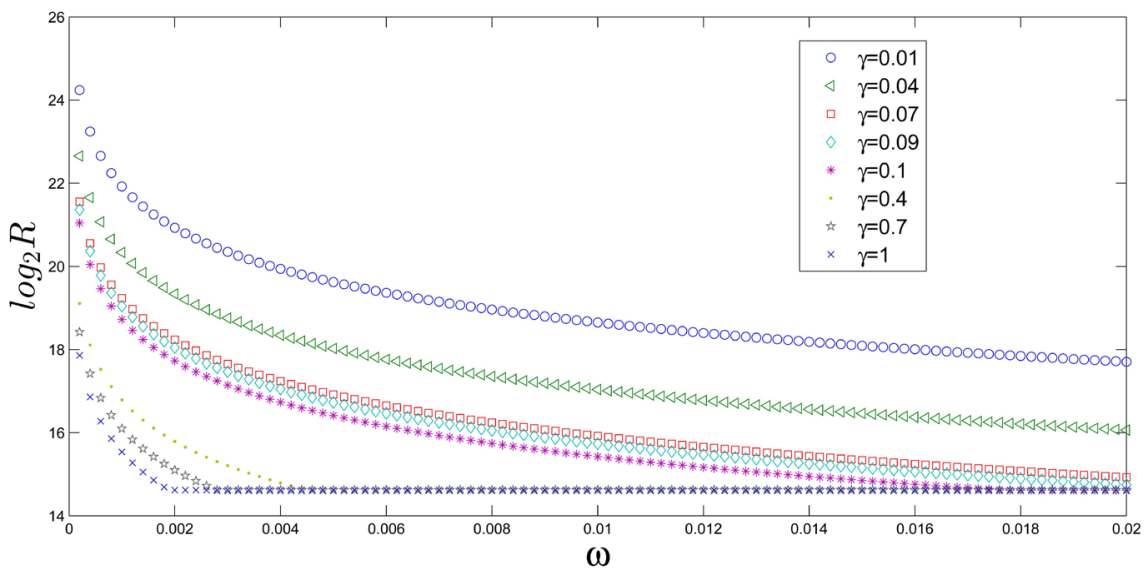

(b)

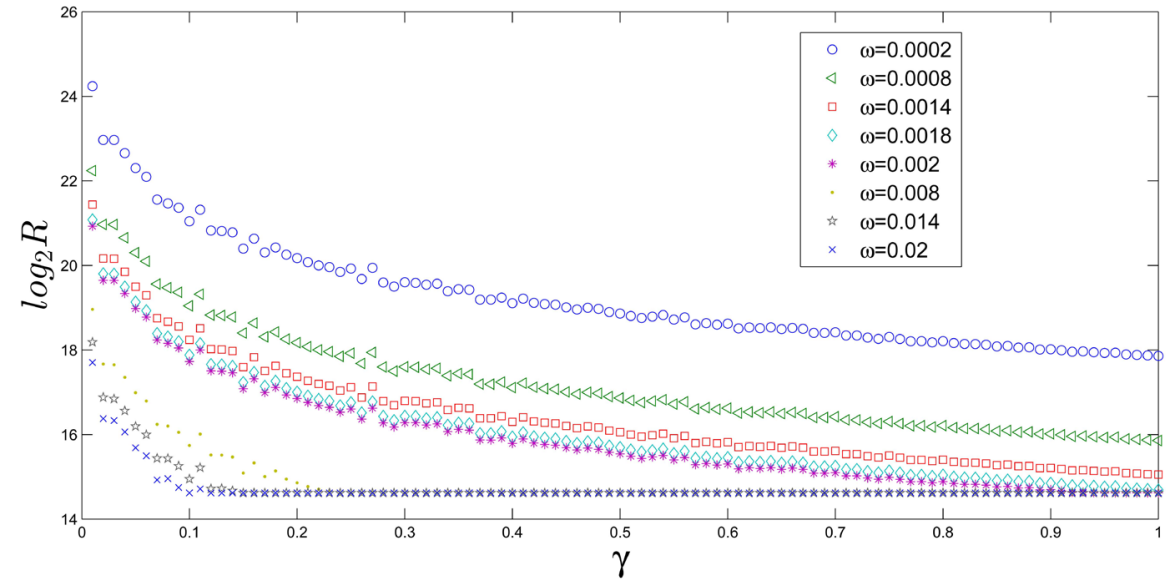

(c)

Figure 11. (a) Phase diagram for $\log _{2} R$ in the two-dimensional parameter space $(\omega, \gamma)$. (b) a $\log _{2} R$ variation diagram of a two-layer WS-cluster ring network with respect to $\omega$. (c) a $\log _{2} R$ variation diagram of a two-layer WS-cluster ring network with respect to $\gamma$.

$\gamma$ values with $\omega$. Figure $7(\mathrm{c})$ is the variation of $\log _{2} R$ of the network corresponding to different $\omega$ values with $\gamma$. In Figure 7 , we use $\log _{2} R$ to 
characterize the synchronizability of the network. We can get the same conclusion in Figure 6: $\omega(\omega \in[0.0002,0.0018])$ is maintained very small, synchronizability is greatly affected by $\gamma$, and the synchronizability of the network will increase as $\gamma$ increases. When $\omega(\omega \in[0.002,0.02])$ is slightly larger, $\gamma$ is getting bigger and bigger, and the synchronizability is not affected by $\gamma \cdot \gamma$ $(\gamma \in[0.01,0.09])$ is maintained very small, synchronizability is greatly affected by $\omega$, and the synchronizability of the network will increase as $\omega$ increases. When $\gamma(\gamma \in[0.1,1])$ is slightly larger, $\omega$ is getting bigger and bigger, and the synchronizability is not affected by $\omega$.

As shown in Figures 8-11, the effects of varying $\omega$ and $\gamma$ on the two-layer WS-cluster ring and the two-layer ER-cluster ring tend to be consistent with the two-layer BA-cluster ring. The simulation results are consistent with the simulation results of two-layer BA-cluster ring.

\section{Conclusion}

In this paper, a two-layer cluster ring network model is established. Through a large number of simulation experiments, the effects of two-layer cluster ring network size variation, interlayer linking weight and interlayer linking fraction change on synchronizability are studied. The experimental results show that such networks are not scalable with respect to synchronizability. Network synchronizability decreases with increasing network size for any cluster structures. Also, the interlayer linking weight and interlayer linking fraction has a significant influence on the network synchronizability. There is an optimum value of the interlayer linking weight in the network, which makes the synchronizability of the network reach the optimum. $\omega$ is maintained very small; synchronizability is greatly affected by $\gamma$, and the synchronizability of the network will increase as $\gamma$ increases. When $\omega$ is slightly larger, $\gamma$ is getting bigger and bigger, and the synchronizability is not affected by $\gamma \cdot \gamma$ is maintained very small, synchronizability is greatly affected by $\omega$, and the synchronizability of the network will increase as $\omega$ increases. When $\gamma$ is slightly larger, $\omega$ is getting bigger and bigger, and the synchronizability is not affected by $\omega$. The smaller interlayer linking weight and the smaller interlayer linking fraction will affect the synchronizability of the two-layer cluster ring network.

\section{Acknowledgements}

This project is supported by National Natural Science Foundation of China (Nos. 61563013, 61663006) and the Natural Science Foundation of Guangxi (No. 2018GXNSFAA138095).

\section{Conflicts of Interest}

The authors declare no conflicts of interest regarding the publication of this paper.

\section{References}

[1] Newman, M.E.J. (2003) The Structure and Function of Complex Networks. SIAM 
Review, 45, 167-256. https://doi.org/10.1137/S003614450342480

[2] Strogatz, S.H. (2001) Exploring Complex Networks. Nature, 410, 268-276. https://doi.org/10.1038/35065725

[3] Albert, R. and Barabsi, A.L. (2002) Statistical Mechanics of Complex Networks. Reviews of Modern Physics, 74, 47-97. https://doi.org/10.1103/RevModPhys.74.47

[4] Newman, M.E.J., Barabsi, A.L. and Watts, D.J. (2006) The Structure and Dynamics of Networks, Vol. 126. Princeton University Press, Princeton, 419-421.

[5] Barabsi, A.L. and Albert, R. (1999) Emergence of Scaling in Random Networks. Science, 286, 509-512. https://doi.org/10.1126/science.286.5439.509

[6] Watts, D.J. and Strogatz, S.H. (1998) Collective Dynamics of "Small-World" Networks. Nature, 393, 440-442. https://doi.org/10.1038/30918

[7] Arenas, A., Diaz-Guilera, A., Kurths, J., et al. (2008) Synchronization in Complex Networks. Physics Reports, 469, 93-153. https://doi.org/10.1016/j.physrep.2008.09.002

[8] Barahona, M. and Pecora, L.M. (2002) Synchronization in Small-World Systems. Physical Review Letters, 89, Article ID: 054101. https://doi.org/10.1103/PhysRevLett.89.054101

[9] Wang, X.F. and Chen, G. (2002) Synchronization in Small-World Dynamical Networks. International Journal of Bifurcation and Chaos, 12, Article ID: 187C92.

[10] Wang, X.F. and Chen, G. (2002) Synchronization in Scale-Free Dynamical Networks: Robustness and Fragility. IEEE Transactions on Circuits and Systems I: Fundamental Theory and Applications, 49, 54-62.

[11] Ichinomiya, T. (2004) Frequency Synchronization in Random Oscillator Network. Physical Review E, 70, Article ID: 026116. https://doi.org/10.1103/PhysRevE.70.026116

[12] Motter, A.E., Zhou, C. and Kurths, J. (2005) Network Synchronization, Diffusion, and the Paradox of Heterogeneity. Physical Review E, 71, Article ID: 016116. https://doi.org/10.1103/PhysRevE.71.016116

[13] Wei, X., Emenheiser, J., Wu, X., et al. (2018) Maximizing Synchronizability of Duplex Networks. Chaos, 28, Article ID: 013110. https://doi.org/10.1063/1.5008955

[14] Lu, J.N., Liu, H. and Chen, J. (2016) Synchronization in Complex Dynamical Networks. Higher Education Press, Beijing.

[15] Lu, J.A., Zhang, Y., Chen, J., et al. (2014) Scalability Analysis of the Synchronizability for Ring or Chain Networks with Dense Clusters. Journal of Statistical Mechanics: Theory and Experiment, P03008.

[16] Xu, M.M., Zhou, J., Lu, J., et al. (2015) Synchronizability of Two-Layer Networks. The European Physical Journal B, 88, 240. https://doi.org/10.1140/epjb/e2015-60330-0

[17] Wei, J., Wu, X., Lu, J.A., et al. (2018) Synchronizability of Duplex Regular Networks. EPL (Europhysics Letters), 120, Article ID: 20005.

[18] Gomez, S., Diaz-Guilera, A., Gomez-Gardenes, J., et al. (2013) Diffusion Dynamics on Multiplex Networks. Physical Review Letters, 110, Article ID: 028701. https://doi.org/10.1103/PhysRevLett.110.028701

[19] Sole-Ribalta, A., De Domenico, M., Kouvaris, N.E., et al. (2013) Spectral Properties of the Laplacian of Multiplex Networks. Physical Review E, 88, Article ID: 032807. https://doi.org/10.1103/PhysRevE.88.032807 